\title{
Gemeinsames Material und Gruppengedächtnis
}

\author{
Schwabe, Gerhard
}

Posted at the Zurich Open Repository and Archive, University of Zurich ZORA URL: https://doi.org/10.5167/uzh-67759

Book Section

Originally published at:

Schwabe, Gerhard (2001). Gemeinsames Material und Gruppengedächtnis. In: Schwabe, Gerhard; Streitz, N; Unland, Rainer. CSCW Kompendium - Lehr- und Handbuch zur computerunterstützten Gruppenarbeit. Berlin / Heidelberg: Springer. 


\title{
Gemeinsames Material und Gruppengedächtnis
}

\author{
Gerhard Schwabe
}

Im Alltagsverständnis wird das mündliche Gespräch als Prototyp für Kommunikation angesehen. Wenn in Veröffentlichungen zur Computerunterstützung der Gruppenarbeit nun davon die Rede ist, daß der Computer die Gruppenkommunikation unterstützt, dann wird das - in dieser Einseitigkeit zu Unrecht - damit gleichgesetzt, daß mündliche Gespräche durch elektronische Kommunikation ersetzt werden (mit allen daraus folgenden Verständnisproblemen und Entfremdungsängsten). Dabei kommen zwei wesentliche Beiträge der CSCW-Forschung zur Unterstützung der Zusammenarbeit zu kurz: Der Computer stellt den Gruppenmitgliedern ein flexibles digitales "gemeinsames Material" zur Verfügung und erlaubt die Ablage von Zwischenergebnissen in einem elektronischen "Gruppengedächtnis". Dieser Beitrag stellt diese beiden Konzepte losgelöst von konkreten Werkzeugen als generische Beiträge von CSCW-Forschung dar.

\section{Gemeinsames Material}

Gemäß dem systemischen Verständnis der Arbeitswissenschaften ist 'Arbeit' die Transformation von 'Material' durch 'arbeitende Menschen' mit Hilfe von 'Arbeitsmitteln' (vgl. das Kapitel zu Arbeitswissenschaften). Bei der Gruppenarbeit sind dabei mehrere Personen gemeinsam tätig, wenn sie beispielsweise gemeinsam ein Stiick Eisen schmieden. Das gemeinsam bearbeite Stück Eisen wird als 'gemeinsames Material' bezeichnet. Das bearbeitete Material braucht nicht stofflich sein, sondern kann auch ideell sein (Steinmüller, 1993, S. 257). Beispielsweise arbeitet ein Autor mit seinem Werkzeug 'Schreibmaschine" sein ideelles Material 'Text'1.

Konventionelle Techniken zur Unterstützung der Gruppenarbeit setzten auf konventionelles gemeinsames Material um Gruppen produktiver zu machen. Dieses gemeinsame Material kann als eine Skizze auf einem Blatt Papier, Stichworte auf einem Flipchart oder als einzelne Worte auf Moderationskärtchen, die an eine Moderationswand gehängt werden, vorliegen. In jedem Fall werden dadurch Gedanken für eine Gruppe gemeinsam erfahrbar und manipulierbar. CSCW-Werkzeuge stellen ein in vieler Hinsicht weitaus flexibleres gemeinsames Material zur Verfügung, als traditionelle Werkzeuge (man denke in erster Näherung nur an die Möglichkeit zur verteilten Bearbeitung des Materials).

Je geeigneter das zur Verfügung stehende gemeinsame Material ist, desto kreativer kann eine Gruppe sein. Dieser Gedankengang von Schrage (1990) soll im folgenden näher ausgeführt werden. Schrage (1990) grenzt über das gemeinsame Material 'Zusammenarbeit' von 'Kommunikation' ab. Klassisch wird Kommunikation als wechselseitige Übertragung von

\footnotetext{
1 Ideeller Inhalt (der Text) und sein Trägermedium (das Stück Papier) sind zwar analytisch voneinander trennbar, aber erstens ist ideeller Inhalt (wie jede Information) immer an ein Trägermedium gebunden und zweitens bestimmt das Trägermedium die Bearbeitungsmöglichkeiten des ideelen Materials. Deshalb wird im Folgenden unter ideellem Material die Kopplung von Trägermedium und getragenem Inhalt verstanden.
} 
Information von Sendern an Empfänger über einen Kanal gedeutet ${ }^{2}$ (Shannon und Weaver, 1949; Watzlawick et al., 1974, S. 23) (vgl. (Abbildung 1).

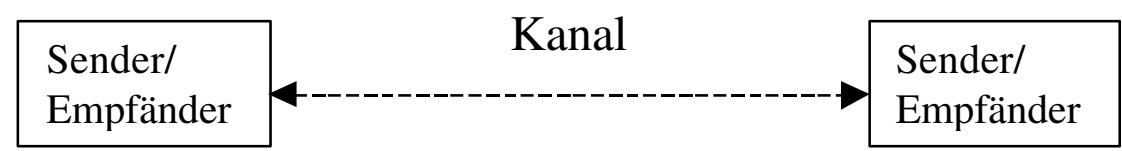

\section{Abbildung 1: Kommunikation}

Bei Kommunikation hat der Kanal keinen Speicher des kommunizierten Inhaltes; nur die Sender und Empfänger speichern den Inhalt. Anders ausgedrückt: Wenn man Information als Modell versteht (Steinmüller, 1993, S. 199), dann gibt es das Modell nur in den Köpfen der Kommunizierenden. Weil es sonst nicht explizit gemacht wird, können die Teilnehmer nur schlecht auf gemeinsame Modellbestandteile Bezug nehmen. Da persönliches Verstehen Modelle subjektiv und das menschliche Gedächtnis unzuverlässig ist, kommt es bei Kommunikation entweder dazu, daß nur auf die in unmittelbarer Vergangenheit übermittelte Information Bezug genommen wird (Schrage, 1990, S. 97 f.), oder es muß sehr viel Aufwand betrieben werden, um die individuellen Modellvorstellungen in Übereinstimmung zu bringen. Für Zusammenarbeit genügt Kommunikation nicht. Sie verlangt einen anderen Ansatz zum Teilen und Schaffen von Information (Schrage, 1990, S. 29) (Abbildung 2).

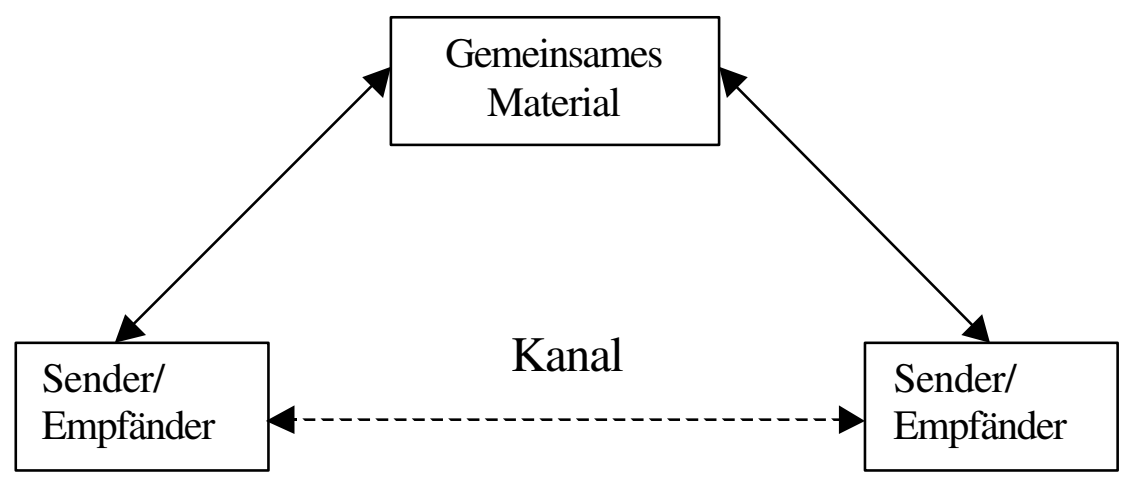

\section{Abbildung 2: Zusammenarbeit (nach (Schrage, 1990, S. 98))}

Bei Zusammenarbeit wird das gemeinsame Modell auf einem gemeinsamen Material - andere nennen es ein gemeinsames Artefakt (Keil-Slawik, 1992; Robinson, 1993) oder gemeinsames externes Gedächtnis (Floyd, 1992, S. 99) - explizit gemacht. Sender und Empfänger kommunizieren weiterhin über einen Kanal. $\mathrm{Zu}$ diesem Kanal tritt ein gemeinsames Material ${ }^{3}$, das als Speicher und Bezugspunkt für die Kommunikationsteilnehmer dient. Gemeinsames Material für die Zusammenarbeit sind beispielsweise Tafeln, Flipcharts, Wandzeitungen mit Kärtchen, dreidimensionale Modelle von Gebäuden in der Architektur, Modelle von Molekülen in der Chemie usw. In den Speicher können die Zusammenarbeitenden Information ablegen und später wieder abrufen. Dadurch können Rückbezüge in die Vergangenheit vereinfacht werden und die Zusammenarbeit gewinnt im Vergleich zur Kommunikation an Tiefe. Anhand des gemeinsamen Materials können die Zusammenarbeitenden ihre Modellvorstellungen verifizieren oder korrigieren. Und sie können das Material gemeinsam bearbeiten, indem sie es umformen, überarbeiten oder aus

2 Kommunikation wird komplexer, wenn man die Beziehungsebene hinzurechnet ((Watzlawick et al., 1974, S. 23; Schulz, 1989, S. 103; Schenk, 1989; Littlejohn, 1992, S. 378 f)). Diese Beziehungsebene hat jedoch für die folgenden Ausführungen über 'gemeinsames Material' keine direkte Bedeutung.

${ }^{3}$ Bei (Schrage, 1990): 'Gemeinsamer Raum', aber Shrage (1990) meint damit das gleiche wie der Autor mit 'gemeinsamen Material'. 
verschiedenen Perspektiven betrachten. Nicht so sehr das Anzeigen von Informationen, sondern der spielerische Umgang mit Ideen und Informationen im gemeinsamen Material ist das Interesse kreativ zusammenarbeitender Menschen (Schrage, 1990, S. 31). Ausgangspunkt von Zusammenarbeit ist häufig ein unfertiges Modell, das gemeinsam von einer Gruppe vervollständigt wird (Schrage, 1990, S. 173).

Gemeinsames Material ist besonders dann nützlich, wenn es vielfältige unterschiedliche Repräsentationen des gleichen Modells darstellen kann, damit das Modell aus unterschiedlichen Perspektiven (z.B. mathematisch, logisch, visuell, strukturell) betrachtet werden kann (Schrage, 1990, S. 155). Die heute üblichen Materialien zur Zusammenarbeit in Gruppen sind in vieler Hinsicht unflexibel, denn sie sind sperrig beim Umformen (bei der Tafel: Auswischen und neu zeichnen/schreiben), sie speichern oft nur wenig Information (Tafeln sind irgendwann voll) und unterstützen einen Wechsel zwischen unterschiedlichen Repräsentationen nicht.

Aus Schrages umfassender Analyse von Zusammenarbeit läßt sich zusammenfassend schließen: Zusammenarbeitende arbeiten an einer gemeinsamen Repräsentation des Problemgegenstands. Diese gemeinsame Repräsentation des Problemgegenstands vollzieht sich auf mehreren Ebenen: Auf einer rein intellektuellen Ebene äußert sie sich in einem wachsenden gemeinsamen Verständnis (Schrage, 1990, S. 7). Die Zusammenarbeitenden nehmen implizit und explizit durch ihre Äußerungen und Beiträge immer wieder Bezug auf dieses 'Gedankengebäude', das gemeinsam geschaffen wurde. Ihnen reicht ein verbaler Bezug auf die gedankliche Repräsentation des Problemgegenstandes bald nicht mehr aus. Sie verwenden Analogien, malen Repräsentationen des Problemgegenstands in die Luft, auf Tafeln oder Papier oder sie bauen physische Modelle (wie z.B. Chemiker Molekülmodelle). Sie verwenden diese Repräsentationen, um den Problemgegenstand erfahrbar, besser verständlich und veränderbar $\mathrm{zu}$ machen. Eine gemeinsame Repräsentation dient dazu, die verschiedenen individuellen Repräsentationen zusammenzufassen und miteinander abzugleichen. Diese gemeinsamen Repräsentationen des Problemgegenstands sind das 'gemeinsame Material' der (geistigen) Gruppenarbeit.

Die Teilnehmer brauchen gemeinsames Material während der Arbeit für drei Zwecke:

- Ein gemeinsames Material dient dazu, ein gemeinsames Verständnis zu schaffen (Schrage, 1990, S. 98) und zu behalten.

- Als Bezugspunkt fokussiert das gemeinsame Material die Arbeit der Mitglieder. Jeder Teilnehmer kann es bearbeiten und hat dadurch am Ergebnis teil.

- Ein gemeinsames Material dient als Gedächtnis. Es speichert das Ergebnis der bisherigen Zusammenarbeit und macht es für alle Zusammenarbeitenden sichtbar. Dadurch können die Teilnehmer feststellen, wo sie in ihrer Arbeit sind, nachvollziehen, wie sie dort hingekommen sind und neue Beiträge in den Kontext des bisher Erreichten einordnen.

"Gemeinsames Material" hat die wissenschaftliche CSCW-Diskussion insbesondere Anfang der 90er Jahre unter dem Stichwort des "Sharings" geprägt. So stand die internationale CSCW-Konferenz 1992 unter dem Stichwort "Sharing Perspectives". Viele Werkzeugentwicklungen hatten zum Ziel die gemeinsame Nutzung von Material möglichst gut zu ermöglichen. Vor diesem Hintergrund sind Gruppenfunktionen wie der Telepointer zum Zeigen in gemeinsamen Dokumenten, das View-Linking zum Synchronisieren von 
Bildschirmausschnitten und das Sperren von Dokumentenausschnitten zu verstehen (siehe das Kapitel zu den Werkzeugen und (Schwabe, 1996)).

\section{Gruppengedächtnis}

Neben der größeren Flexibilität bei der Handhabung während der Arbeit ist der vereinfachte Rückgriff auf alte Ergebnisse in einem digitalen "Gruppengedächtnis" ein wesentlicher Unterschied zwischen konventioneller und computerunterstuitzter Zusammenarbeit.

In Besprechungen, Sitzungen und Workshops werden viele Informationen generiert, die für Organisationen von großer Bedeutung sind. Da Menschen sich nicht besonders gut an die Vergangenheit erinnern (Huber, 1990) und sich besonders schlecht die Gründe für Entscheidungen merken (Walsh\&Ungson, 1990), sind sie nur sehr unzuverlässige Speicher für Informationen aus Sitzungen. Die meisten Aussagen in einer konventionellen Sitzung sind flüchtig, sei es weil sie nur ausgesprochen und nicht protokolliert werden oder sei es, weil sie zwar protokolliert werden, aber nur auf einem flüchtigen Medium (z.B. einer Tafel). Bei der computerunterstützten Zusammenarbeit werden viele Informationen digital abgelegt. Somit kann auch später auf sie zurückgegriffen werden, wenn diese geeignet abgelegt sind und geeignete Werkzeuge zum Informationsabruf zur Verfügung stehen. Dies kommt der Natur von Gruppenarbeit in Unternehmen entgegen. Sitzungen und Besprechungen finden nur selten in Isolation statt; meist sind sie in einen längeren Aktivitätsfluß eingebettet, der auch Phasen der Einzelarbeit und die Zusammenarbeit in unterschiedlichen Konstellationen von Untergruppen umfaßt (Krcmar, 1989; Morrison et al., 1992; Morrison, 1993). Aus der Perspektive einer Person stellt eine bestimmte Gruppe in der Regel auch nur einen von mehreren Arbeitszusammenhängen dar; für andere Aufgaben ist er in andere Gruppen eingebunden. Es kann ihm dabei Nutzen bringen, Informationen aus dem einen Gruppenzusammenhang in einem anderen Zusammenhang $\mathrm{zu}$ nutzen. Dies kann ein elektronisches Gruppengedächtnis ermöglichen.

Die wissenschaftliche Diskussion zu einem digitalen Gruppengedächtnis ist in die Diskussion zu einem Organisationsgedächtnis (Organizational Memory) eingebettet. Amerikanische Forscher verstehen unter Organizational Memory dabei sowohl die "gespeicherte Information aus der Geschichte einer Organisation, die für aktuelle Probleme zunutze gemacht werden können" (Walsh\&Ungson, 1991, S. 61) (also die gespeicherte Information selbst) als auch als "das Mittel, durch das Wissen aus der Vergangenheit für aktuelle Aktivitäten zur Wirkung gebracht werden kann und dadurch $\mathrm{zu}$ einem höheren oder niedrigerem Niveau von organisatorischer Effektivität führt"(Stein\&Zwass, 1995, S. 89). Unter Organizational Memory wird also sowohl gespeicherte Information als auch umfassender als gespeicherter Zustand eines Geflechts aus "Artefakten und Personen, Speicher und Speicherverarbeitung" (Ackerman\&Halverson, 1998, S. 47) verstanden. Eine Organisation benötigt Information aus der Vergangenheit, um laufende Projekte zu unterstïtzen, seine Aktivitäten zu koordinieren, als Modell für zukünftige Aktivitäten, um vergangene Entscheidungen zu erklären, um prognostizieren $\mathrm{zu}$ können, schädlichen Mythen $\mathrm{zu}$ begegnen, als Ausgangspunkt für Veränderungen und um Fehlerwiederholung $\mathrm{zu}$ vermeiden (Schwabe, 1995). Diese Funktionen eines Organisationsgedächtnisses können den einen nutzen und den anderen schaden. Beispielsweise ist die Nachvollziehbarkeit eines Softwareentwurfs (des sogenannten Design Rationale (Conklin\&Begemann, 1988; Conklin, 1993) für spätere Modifikationen der Software nützlich. Allerdings wird dann in der Regel auch die Verantwortung für 
Fehlentscheidungen dokumentiert. Dies kann soweit gehen, daß auf der Basis dieser archivierten Informationen Unternehmen für Fehler bei der Softwareerstellung verklagt werden können. Somit besteht bei einem Gruppengedächtnis die häufig bei Groupware zu beobachtende ungleiche Verteilung von Kosten und Nutzen (Grudin, 1988).

Aus Sicht des Softwaredesigners stellt das Konzept des Gruppengedächtnisses die Brücke von CSCW-Werkzeugen zu Datenbanken her. Die Bedeutung der Memory-Funktion wird daran deutlich, daß mit Lotus Notes die am weitesten verbreitete kommerzielle Groupware Dokumentendatenbanken zum Kern der Unterstützung macht. Die Herausforderungen bei der Gestaltung von computerunterstützten Gruppengedächtnissen sind dabei (Schwabe, 1999):

1. Die Speicherung von semantisch reichen Informationen. Dies bedeutet insbesondere, daß möglichst viel Information über den Arbeitskontext abgelegt wird, z.B. die an einer gemeinsamen Aktivität beteiligten Personen, das Datum, der Bezug eines Zwischenergebnisses zu einem Tagesordnungspunkt und die inhaltliche Verknüpfung von Einzelergebnissen. Da diese Informationen in Gruppen ohnehin kommuniziert und damit in höherem Maße explizit gemacht werden müssen, als bei der Einzelarbeit, bieten sich zahlreiche Ansatzpunkte für die Speicherung von Kontextinformationen.

2. Diese Informationen müssen aber ohne größeren Aufwand im Hintergrund erfaßt und aufgearbeitet werden, damit ein elektronisches Gruppengedächtnis Akzeptanz findet.

3. Die Informationen müssen für die einzelnen Gruppenmitglieder einfach zugänglich und wartbar sein. Zu der Wartung gehört auch das "Vergessen" (Landry, 1999) von Informationen.

4. Die Informationen der einzelnen Gruppen müssen mit anderen Informationen (von weiteren Gruppen oder sonstigen Quellen) aus der Organisation integriert werden. Dies erfordert eine Einigung auf Standards zumindest innerhalb einer Organisation.

Ein elektronisches Gruppengedächtnis wirft dann erhebliche Datenschutzprobleme (inbesondere Schutz der Privatsphäre) auf, wenn es in ein umfassendes elektronisches Organisationsgedächtnis integriert ist. Deshalb sollten Mechanismen zur Verfügung stehen, die automatisch über die Zeit oder gezielt auf Anforderung Kontextinformationen unschärfer werden lassen: Z.B. kann über die Zeit möglicherweise auf die Speicherung individueller Autorenschaft von Beiträgen verzichtet werden, solange die Gruppe oder Abteilung als Kontextinformation gespeichert wird. Oder die Teilnehmer können schon während der Kooperation eine anonyme Speicherung von Informationen vereinbaren.

\section{Literatur}

Ackermann, M.; Halverson, C. (1998): Considering an Organization's memory. In: Proceedings of CSCW 98. ACM Press: New York, 39-48.

Conklin, J. (1993). Capturing Organizational Memory. In: Becker, R.: Readings in Groupware and CSCW. Morgan Kaufmann: San Mateo, 561-565.

Conklin, J., Begemann, M. (1988). gIBIS: A hypertext tool for exploratory policy discussion. In: Proceedings of $C S C W^{\prime} 88$, September 26 to 28, Portland, Oregon, 140-152.

Floyd, C. (1992). Software development as reality construction. In: Floyd, C. et al.: Software development and reality construction. Springer: Berlin u.a., 86 - 100.

Grudin, J. (1988). Why CSCW-Applications fail: Problems in the design and evaluation of organizational interfaces. In: Proceedings of the CSCW 1988, Portland, 85-93. 
Huber, G. (1990). A Theory of the Effects of Advanced Information Technologies on Organizational Design, Intelligence, and Decision Making. Academy of Management Review, 15,1,47-71.

Keil-Slawik, R. (1992). Artifacts in software design. In: Floyd, C. et al.: Software development and reality construction. Springer: Berlin u.a., 168 -188.

Krcmar, H. (1989). Considerations for a framework for CATeam research. In: Proceedings of the First European Conference on Computer Supported Co-operative Work, 13th - 15th September), London , 421-435.

Landry, J. (1999). Forgetful or bad memory. In: Hawaii International Conference on System Sciences 1999 (HICSS99), CD-ROM, 8 pages.

Morrison J. (1993). Team memory: Information management for business teams. In: Proceedings of the 26th Hawaii International Conference on Systems Sciences Jan. 5-8, Wailea, Hawaii.

Morrison, J., Morrsion, M., Vogel, D. (1992). Software to support business teams. In: Group Decision and Negotiation, Vol. 1, Nr. 2, 91 - 115.

Robinson, M. (1993). Keyracks and computers: An introduction to "common artefacts" in computer supported cooperative Work". In: Wirtschaftsinformatik, Vol. 35, Nr. 2 April , 157 166.

Schrage, M. (1990). Shared minds - the new technologies of collaboration. Random House: New York.

Stein, E. ,\& Zwass, V. (1995). Actualizing Organizational Memory with Information Systems. Information Systems Research, 6, 85-117.

Steinmüller, W. (1993): Informationstechnologie und Gesellschaft, Wissenschaftliche Buchgesellschaft, Darmstadt 1993.

Schwabe, G. (1995). Objekte der Gruppenarbeit - ein Konzept für das Computer Aided Team. Gabler: Wiesbaden.

Schwabe, G. (1999). Providing for Organizational Memory in Computer Supported Meetings. In: Journal of Organizational Computing and Electronic Commerce, Nr. 2\&3, 151169.

Schwabe, G., Krcmar, H. (1996). CSCW Werkzeuge. In: Wirtschaftsinformatik, Vol. 38, Nr. 2, 209-224.

Shannon, C., Weaver, W. (1949). The mathmatical theory of communication. University of Illinois Press: Urbana.

Steinmüller, W. (1993). Informationstechnologie und Gesellschaft. Wissenschaftliche Buchgesellschaft: Darmstadt.

Walsh, J.P.,\& Ungson G.R. (January 1991). Organizational Memory. Academy of Management Review,16,1,57-91.

Watzlawick, P., Beavin, J., Jackson, D. (1974). Menschliche Kommunikation, 4. Auflage. Hans Huber: Bern et al. 